\title{
TENSIONES DE CRECIMIENTO EN Eucalytpus dunnii Maiden.: PARÁMETROS DENDROMÉTRICOS Y ANATOMÍA DE LA MADERA ${ }^{1}$
}

\author{
Silvia Monteoliva² e Mariano Hernández ${ }^{3}$
}

\begin{abstract}
RESUMEN - El objetivo del trabajo fue determinar el efecto de los parámetros dendrométricos de los árboles y la anatomía de la madera sobre las tensiones de crecimiento en tres procedencias de Eucalytpus dunnii Maiden. Se muestrearon 15 árboles de E. dunnii de 19 años en Corrientes, Argentina (28 26' 23,7' S 58 59' 47,7" $\mathrm{O}$ a una altitud de $65 \mathrm{~m}$ ). Se determinaron tensiones de crecimiento a través de dos métodos: indicador de tensiones de crecimiento (GSI, CIRAD-Forêt) e índice de rajado en rollizo (IR). Se midió la altura total del árbol, el diámetro (DAP), la relación altura/diámetro, un índice de competencia individual y algunas propiedades anatómicas (espesor de pared fibras, área de pared ocupada, diámetro y frecuencia de vasos). Las procedencias no fueron una fuente significativa de variación para ninguna de las variables. El GSI e IR mostraron magnitudes más bajas que la citadas para la especie (GSI: 0,039-0,137 mm e IR: 0,241-1,866 \%). Los valores del espesor de pared de las fibras $(3,39 \mu \mathrm{m} \pm 0,35)$ son un poco elevados con respecto a los valores esperados lo que podría indicar presencia de leño de tensión. La altura del árbol y la competencia individual no presentaron una asociación significativa con las tensiones de crecimiento. El DAP y la relación AT/DAP tuvieron una relación significativa con el IR solamente $\left(\mathrm{R}^{2}=0,42\right.$ para AT/DAP y 0,27 para DAP). Las relaciones entre tensiones y las variables anatómicas cuantitativas no fueron significativas en ningún caso.
\end{abstract}

Palabras-clave: Anatomía de madera; GSI; Índice de rajado.

\section{TENSÕES DE CRESCIMENTO EM Eucalytpus dunnii Maiden.: PARÂMETROS DENDROMÉTRICOS E ANATOMIA DA MADEIRA}

\begin{abstract}
RESUMO - O objetivo deste estudo foi determinar o efeito dos parâmetros dendrométricos das árvores e da anatomia da madeira sobre a tensão de crescimento em três procedências de Eucalytpus dunnii. Quinze árvores de 19 anos foram amostradas de Corrientes, Argentina (28 26 23, $7^{\prime \prime} S 58^{\circ} 59^{\prime} 47,7^{\prime \prime} \mathrm{O}$, a uma altitude de $65 \mathrm{~m}$ ). Tensões de crescimento foram determinadas por dois métodos: indicador da tensão de crescimento (GSI) e índice rachado em tora (IR). Mediram-se a altura total da árvore, o diâmetro (DAP), a relação altura/diâmetro, o indice de competência individual e algumas propriedades anatômicas (espessura da parede da fibra, na área da parede ocupada e do diâmetro dos vasos e sua frequência). As procedências não foram fontes significativas de variação para qualquer uma das variáveis. O GSI e o IR foram de magnitude menor do que o citado para essa espécie (GSI: ,039-,137 mm e IR: 0,241-1,866\%). Os valores da espessura das paredes das fibras $(3,39 \pm 0,35 \mu \mathrm{m})$ foram levemente elevados em relação ao esperado, o que indica a presença de madeira de tensão. A altura da árvore e a competência individual não apresentaram associação significativa com as tensões de crescimento. O DAP e AT/DAP tiveram relação significativa com $I R\left(R^{2}=0,42\right.$ para AT/ DAP e 0,27 para DAP). As relações entre tensões e variáveis anatômicas quantitativas não foram significativas.
\end{abstract}

Palabras-chave: Anatomia da madeira; GSI; Índice rachado.

\footnotetext{
${ }^{1}$ Recebido em 28.05.2013 aceito para publicação em 27.05.2014.

${ }^{2}$ Facultad Cs. Agrarias y Forestales, UNLP. INFIVE-CONICET. E-mail: <smonteoliva@yahoo.com.ar>.

${ }^{3}$ Instituto Nacional de Tecnología Agropecuaria (INTA), EEA Bella Vista, Corrientes. Argentina. E-mail:

$<$ mahernandez@correo.inta.gov.ar>.
} 


\section{INTRODUCCIÓN}

El uso principal de la especie Eucalyptus dunnii Maiden a nivel mundial es como materia prima para la industria de la pulpa y el papel. En la industria de la transformación mecánica se la utiliza en la elaboración de pisos, fabricación de piezas estructurales en la construcción o en la elaboración de chapas para tableros compensados (DICKSON et al., 2003). Es apta para la fabricación de paneles LVL, cabos de herramientas, elementos deportivos, entre otros productos forestales (PEREYRA, 2000; PEDROSA, 2003). Sin embargo, su uso como madera aserrada reportó problemas tecnológicos vinculados a tensiones de crecimiento, elevada retractabilidad, alto gradiente de humedad, secado lento, rajaduras superficiales y colapso durante el secado (SEVERO; TOMASELLI, 2001).

El término tensiones de crecimiento fue propuesto para designar a las fuerzas internas presentes en el leño de los árboles en pie. Las tensiones de crecimiento resultan de la superposición de dos tipos de tensiones: las tensiones de soporte y las tensiones de maduración (GERARD, 1994). El proceso de polimerización de la pared celular conlleva a una mayor rigidez e induce pequeñas deformaciones, usualmente contracciones axiales y expansiones transversales de la célula. La mayor parte de estas deformaciones son impedidas por las paredes rígidas de células formadas en años anteriores. Esto provoca esfuerzos de tensión en sentido longitudinal y esfuerzos de compresión en sentido tangencial (THIBAUT; GRIL, 2003). La magnitud de estas fuerzas es muy variable según la dirección que se considere, aunque la mayor parte de los trabajos abordan el estudio de las tensiones de crecimiento solo en dirección axial (KUBLER, 1987; VIGNOTE et al., 1996).

Cuando el árbol es apeado, se produce la liberación de las tensiones de crecimiento a partir de la expansión longitudinal del centro sometido a compresión y de la contracción de la periferia que estaba sometida a fuerzas de tensión. Esto provoca que se produzcan rajaduras en el leño que dan como resultado el estrellado observable en las caras transversales del rollizo, además de otros defectos como deformaciones o alabeos que disminuyen la calidad de las tablas aserradas (GERARD, 1992; VIGNOTE et al., 1996; RAYMOND et al., 2004; VALENCIA et al., 2011).

Múltiples serían los factores que afectan los niveles de tensiones de crecimiento de los árboles en una plantación, pero su influencia no se encuentra del todo clara. Se pueden nombrar la edad, tamaño de los árboles, efecto de los vientos, raleos, podas, densidad de plantación, estado de competencia entre árboles (KUBLER, 1987; VIGNOTE et al., 1996). Para la especie en estudio E. dunnii, Murphy et al. (2005) han publicado la influencia de la relación altura/diámetro sobre las tensiones de crecimiento en un estudio sobre 15 procedencias de 9 años en Australia. También se ha reportado la influencia del tamaño de los árboles (medidos como diámetro y volumen) y la edad sobre las tensiones en estudios realizados en España sobre E. globulus. La asociación establecida por regresiones resulta inversa, es decir que a mayor edad y diámetro disminuyen las tensiones medidas como GSI. La altura total del árbol y el volumen de copa no afectaron las medidas de GSI (VIGNOTE et al., 1996).

Según la bibliografía, se han establecido correlaciones entre tensiones y parámetros anatómicos como leño de tensión, ángulo microfibrillar, frecuencia de vasos y con la composición química (contenidos de celulosa y lignina). Los resultados reportados para distintas especies de angiospermas son dispares ya que no siempre se encuentran correlaciones significativas entre estas variables (OKUYAMA et al., 1993; YOSHIDA et al., 2000, 2002; CLAIR et al., 2003; RUELLE et al., 2006; GACITUA et al., 2007; FANG et al., 2008). Para las tensiones de crecimiento en el género Eucalyptus se han reportado efectos del leño de tensión pero sin presencia de la capa $\mathrm{G}$, del contenido de lignina y las proporciones de unidades G o S y de la medida del ángulo microfibrillar en la pared secundaria (BAILLERES et al., 1995; RAYMOND et al., 2004). También se han vinculado con el diámetro y frecuencia de vasos (RUELLE et al., 2006; GACITUA et al., 2007).

Asimismo, las tensiones de crecimiento tienen un control genético y la ausencia de severas rajaduras es un importante criterio de selección en los programas de mejoramiento genético forestal (RAYMOND et al., 2004; MALAN, 2008). Se han reportado diferencias significativas en las tensiones de crecimiento según la procedencia en E. globulus y E. dunnii (RAYMOND et al., 2004; MURPHY et al., 2005), sin embargo otros autores determinaron que la procedencia no influyó en las determinaciones de GSI para la primera especie (VIGNOTE et al., 1996; YANG et al., 2002). Por lo tanto, identificar a los materiales más aptos, evaluar su comportamiento en el proceso de transformación mecánica y profundizar en las causas y relaciones vinculadas

Revista Árvore, Viçosa-MG, v.38, n.4, p.755-763, 2014

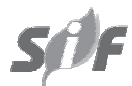


a este problema, son los primeros pasos en la búsqueda para disminuir el efecto negativo de las tensiones de crecimiento en el uso de esta especie como madera aserrada.

El objetivo del trabajo fue determinar el efecto de los parámetros dendrométricos de los árboles y la anatomía de la madera sobre las tensiones de crecimiento en tres procedencias de Eucalytpus dunnii.

\section{MATERIALES Y MÉTODOS}

Se muestreó un ensayo de E. dunnii de 19 años situado en la localidad de Bella Vista, Provincia de Corrientes, Argentina. Su ubicación geográfica exacta corresponde a las coordenadas $28^{\circ} 26^{\prime} 23,7^{\prime \prime} \mathrm{S} 58^{\circ} 59^{\prime}$ 47,7" O a una altitud de 65 m.s.n.m. Fue plantado en el año 1992 en un albardón cercano al río Paraná, en suelos arenosos profundos en un sitio en los que no existen evidencias de disturbios antrópicos importantes anteriores a su establecimiento. El clima de la región es húmedo y mesotermal, correspondiendo a la clasificación de Thornthwite C2 B'4 ra' (Castro et al., 1991), con $1218 \mathrm{~mm}$ de precipitación media anual, $20,9^{\circ} \mathrm{C}$ de temperatura media anual $\left(26^{\circ} \mathrm{C}\right.$ de temperatura máxima y $15,7^{\circ} \mathrm{C}$ de mínima anual) y 328 días como periodo libre de heladas.

El material muestreado forma parte de una red de ensayos de procedencias y familias distribuidos en distintos sitios de la Mesopotamia Argentina. El material genético de los ensayos fue recolectado de poblaciones naturales ubicadas en el estado de Nueva Gales del Sur, Australia (Tabla 1). El ensayo fue plantado en un diseño de bloques completos al azar con 15 repeticiones conformado por parcelas de una sola planta con un distanciamiento de $3 \mathrm{~m}$ x $3 \mathrm{~m}$. No se realizaron intervenciones silvícolas en el ensayo.

Se muestrearon 15 árboles (5 por procedencia) pertenecientes al estrato superior del bosque. Para cada árbol se midió con forcípula e hipsómetro el diámetro a la altura del pecho (DAP) y la altura total (AT). Con estos valores se calculó la relación AT/DAP.
Se calculó para cada árbol muestreado un índice de competencia individual (CI) que considera que el efecto de la competencia que ejercen los árboles que lo rodean aumenta cuando se incrementa el tamaño de estos últimos y cuando se reduce la distancia que los separa del árbol analizado.

$$
C I_{i}=\sum_{i \neq j} \frac{d j}{d i} x \frac{1}{D i j}
$$

CIi $=$ Valor del índice de Hegyi para el árbol i.

di = Diámetro normal del árbol de referencia i.

$\mathrm{d}=$ Diámetro normal del competidor $\mathrm{j}(\mathrm{j} \neq \mathrm{i})$.

Dij = Distancia entre el árbol i y el competidor $\mathrm{j}$.

Sobre el material de estudio se realizaron determinaciones de tensiones de crecimiento a través de dos métodos: indicador de tensiones de crecimiento (GSI) e índice de rajado en rollizo (IR).

\subsection{Determinación del indicador de tensiones de crecimiento (GSI)}

Se aplicó el método CIRAD-Forêt (YANG et al., 2005) para la determinación de los valores GSI. En los árboles en pie se abrió una ventana rectangular sobre la corteza de $2,5 \mathrm{~cm}$ de ancho por $9 \mathrm{~cm}$ de alto la cual dejó expuesta la superficie del cambium. Luego se colocó el extensómetro, se realizó la perforación con un taladro manual y se registró el valor de desplazamiento de los pines. Las mediciones se realizaron a una altura de 1,3 m. en las posiciones cardinales Norte, Este, Sur y Oeste. Con los valores de desplazamiento obtenidos para las cuatro posiciones cardinales se calculó el valor GSI promedio por árbol.

\subsection{Determinación del índice de rajado en rollizo}

Se apearon los árboles y se cortó un rollizo de $2 \mathrm{~m}$ de longitud por encima de los 1,3 $\mathrm{m}$. Inmediatamente abatidos los árboles se colocaron bolsas plásticas sobre los extremos de los rollizos. Durante cinco días los rollizos permanecieron en el ensayo con los extremos

Tabla 1 - Procedencias de E. dunnii muestreadas.

Tabela 1 - Procedências de E. dunnii amostrados.

\begin{tabular}{ll}
\hline Procedencias & Localización \\
\hline Death Horse (DH) & Lat: $28^{\circ} 25^{\prime} \mathrm{S}-$ Long: $152^{\circ} 20^{\prime}$ E Alt: $600-700 \mathrm{~m}$. \\
Boomi Creek (BC) & Lat: $28^{\circ} 25^{\prime}$ S - Long: $152^{\circ} 41^{\prime}$ E Alt: $300 \mathrm{~m}$. \\
Oaky Creek (OC) & Lat: $28^{\circ} 36^{\prime}$ S - Long: $152^{\circ} 31^{\prime}$ E Alt: $520 \mathrm{~m}$. \\
\hline
\end{tabular}

Revista Árvore, Viçosa-MG, v.38, n.4, p.755-763, 2014 
cubiertos. Al quinto día se quitaron las bolsas y se sacó una fotografía digital de cada una de las dos caras transversales de los rollizos junto a la escala de una forcípula. Se volvió a cubrir cada rollizo con las bolsas hasta su traslado al aserradero.

Las fotografías de las caras transversales de los rollizos fueron analizadas en gabinete con el software de medición Micrometrics SE Premium. En cada fotografía se tomaron las mediciones necesarias para poder calcular un índice de rajado para cada rollizo según la fórmula 2 :

$$
I R=200 \times \frac{\sum_{i=1}^{n} a_{i} L_{i}}{\pi D_{i}^{2}}
$$

IR: Índice de rajado para los extremos del rollizo (\%).

$a \mathrm{i}$ : Abertura máxima de la enésima rajadura ( $\mathrm{mm})$.

Li: Longitud de la enésima rajadura ( $\mathrm{mm})$.

Di: Diámetro sin corteza medio de la sección transversal del rollizo considerado $(\mathrm{mm})$.

\subsection{Anatomía madera}

Luego de apeado el árbol se cortó una rodaja a la altura de las determinaciones de GSI $(1,3 \mathrm{~m})$. Sobre dicha rodaja se tomó una probeta cúbica en el radio norte de $2 \mathrm{~cm}$ de lado, contigua a la ventana abierta sobre la corteza en el punto de determinación de GSI.

Se realizaron cortes transversales con micrótomo (de 20um) y se tiñeron con safranina al 1\%. Se tomaron imágenes con una cámara digital (Olympus DP71, Japón) asociada a un microscopio óptico Olympus BX50. Sobre las imágenes (40x y 200x) se midieron espesor doble de pared ( $\mathrm{n}=100$ por árbol), área de pared ocupada $(\mathrm{n}=10$ imágenes capturadas por árbol, según ecuación 3), diámetro de vasos (n=100 por árbol) y número de vasos por $\mathrm{mm}^{2}$ ( $\mathrm{n}=7$ campos por árbol). Las medidas se realizaron con el software Image Tool versión libre.

$$
A P O(\%)=\frac{A T-(A L V+A L F+A P R) \cdot 100}{A T}
$$

APO: área de pared ocupada (\%)

AT: área total de la imagen capturada

ALV: área de lumen de vasos

ALF: área de lumen de fibras

APR: área de parénquima radial

\subsection{Análisis estadístico de datos}

Para poder utilizar las diferentes procedencias como un mismo conjunto se realizó el análisis de la varianza de las variables dependientes diámetro a la altura de pecho (DAP), altura total del árbol (AT), relación AT/ DAP, competencia individual (CI), espesor de pared de las fibras, área de pared ocupada, diámetro y frecuencia de vasos, IR y GSI. Se estableció la hipótesis nula de igualdad de las medias entre las procedencias estudiadas. Se utilizó el siguiente modelo lineal aditivo:

$$
Y_{i j}=\mu+\tau_{i}+\varepsilon_{i j}
$$

$Y_{i j}$ : Observación de la procedencia i repetición j $\mu$ : Media general

$\tau$ : Efecto de la procedencia $\mathrm{i}$

$\varepsilon_{i j}:$ Error experimental

Para conocer el efecto de las variables diámetro a la altura de pecho (DAP), altura total del árbol (AT), relación AT/DAP, competencia individual (CI), espesor de pared de las fibras, área de pared ocupada, diámetro y frecuencia de vasos, sobre las variables dependientes GSI e IR se planteó un análisis de regresión lineal simple.

\section{RESULTADOS}

Los resultados del análisis de la varianza indicaron que las procedencias no fueron una fuente significativa de variación para ninguna de las variables de crecimiento, anatómicas o de tensiones ( $\mathrm{p}>0,05)$. Por lo tanto los resultados de todos los árboles se analizaron como un conjunto $(n=15)$.

Los caracteres anatómicos cuantitativos indicaron para esta especie que la madera presentó vasos de gran diámetro y poco numerosos y un área de pared ocupada cercanos al 70\%. Los valores del espesor de pared de las fibras (Tabla 2, Figura 1) son un poco elevados. Se observó además la presencia de fibras de aspecto redondeado y espacios intercelulares (Figura 1, flecha), lo que podría indicar la presencia de leño de tensión, aunque ésto no fue cuantificado.

Los resultados de las tensiones de crecimiento, medidos como GSI e IR, mostraron la variabilidad característica que se presenta con las tensiones en la bibliografía, se destaca la gran dispersión que presenta el IR generado básicamente en la procedencia BC. La mayor parte de los datos de GSI se ubica por encima 
Tabla 2 - Valores medios ( \pm desvió estándar) de todas las variables analizadas por procedencia. Tabela 2 - Médias ( \pm desvio-padrão) de todas as variáveis analisadas pela procedências.

\begin{tabular}{lcccc}
\hline & $\mathrm{DH}(\mathrm{n}=5)$ & $\mathrm{OC}(\mathrm{n}=5)$ & $\mathrm{BC}(\mathrm{n}=5)$ & Promedio(n=15) \\
\hline Diámetro (DAP) cm & $26,7( \pm 1,04)$ & $30,2( \pm 2,15)$ & $27,1( \pm 3,56)$ & $28,0( \pm 2,8)$ \\
Altura total (AT) m & $27,3( \pm 4,4)$ & $30,1( \pm 0,6)$ & $26,9( \pm 1,5)$ & $28,1( \pm 2,9)$ \\
AT/DAP & $101,8( \pm 14)$ & $100,3( \pm 6)$ & $101,1( \pm 18)$ & $101,1( \pm 13)$ \\
Competencia individual & $0,91( \pm 0,38)$ & $0,68( \pm 0,36)$ & $1,02( \pm 0,36)$ & $0,87( \pm 0,37)$ \\
Espesor pared fibras $\mu \mathrm{m}$ & $3,38( \pm 0,37)$ & $3,28( \pm 0,43)$ & $3,52( \pm 0,27)$ & $3,39( \pm 0,35)$ \\
Área pared ocupada \% & $68( \pm 6)$ & $67( \pm 3)$ & $69( \pm 4)$ & $68( \pm 4)$ \\
Diámetro vasos $\mu \mathrm{m}$ & $117,5( \pm 26,2)$ & $113,4( \pm 11,0)$ & $109,1( \pm 8,1)$ & $113,3( \pm 16,2)$ \\
Frecuencia vasos $\mathrm{n}^{\circ} . \mathrm{mm}^{-2}$ & $8( \pm 3)$ & $8( \pm 2)$ & $8( \pm 2)$ & $8( \pm 2)$ \\
IR \% & $0,45( \pm 0,14)$ & $0,35( \pm 0,12)$ & $0,58( \pm 0,72)$ & $0,45( \pm 0,41)$ \\
GSI mm & $0,081( \pm 0,03)$ & $0,071( \pm 0,01)$ & $0,08( \pm 0,04)$ & $0,081( \pm 0,03)$ \\
\hline
\end{tabular}

IR: índice de rajado, GSI: indicador de tensiones de crecimiento, DH, OC y BC: procedencias.

IR: indice rachado em tora, GSI: indicador da tensão de crescimento, DH, OC y BC: procedencias.

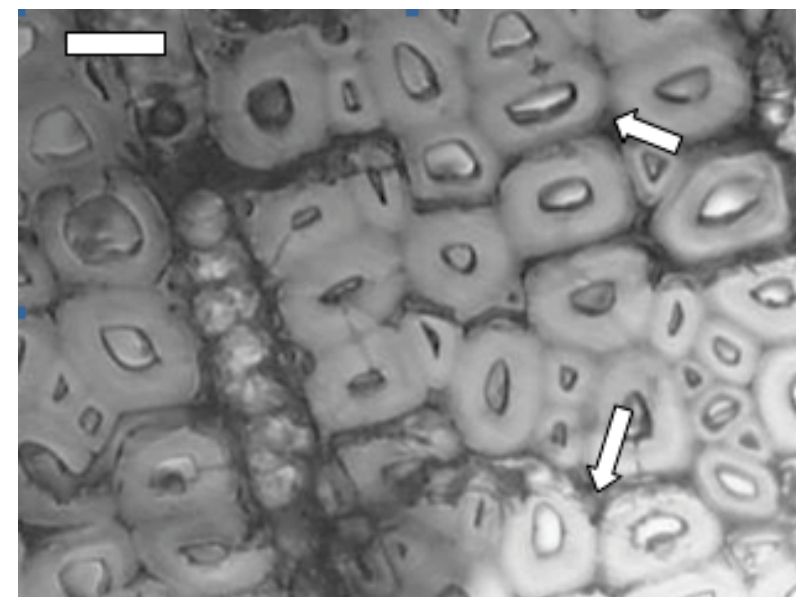

Figura 1 - Corte transversal. Presencia de fibras de paredes muy gruesas. Fibras con bordes redondeados y espacios intercelulares (flechas). Escala: $10 \mu \mathrm{m}$.

Figura 1 - Corte Transversal. Presença de fibras de paredes espessas. Fibras com arestas arredondadas e espaços intercelulares (setas). Escala: $10 \mu \mathrm{m}$.

de los 0,05 mm y alcanza un máximo de 0,14 $\mathrm{mm}$. Para el IR solo un árbol alcanzó un valor superior al 1\%, el resto de los árboles oscilaron entre $0,20 \%$ y $0,60 \%$.

\subsection{Relaciones entre variables}

La altura y la competencia individual no presentaron una asociación significativa con las tensiones de crecimiento según los modelos de regresión ( $p>0,05)$. El DAP y la relación AT/DAP tuvieron una relación significativa con el IR solamente (Figuras 2 y 3 ). Los bajos valores de coeficiente de determinación muestran que el tamaño de los árboles no explica la variabilidad encontrada en las tensiones de crecimiento estimadas con el IR.

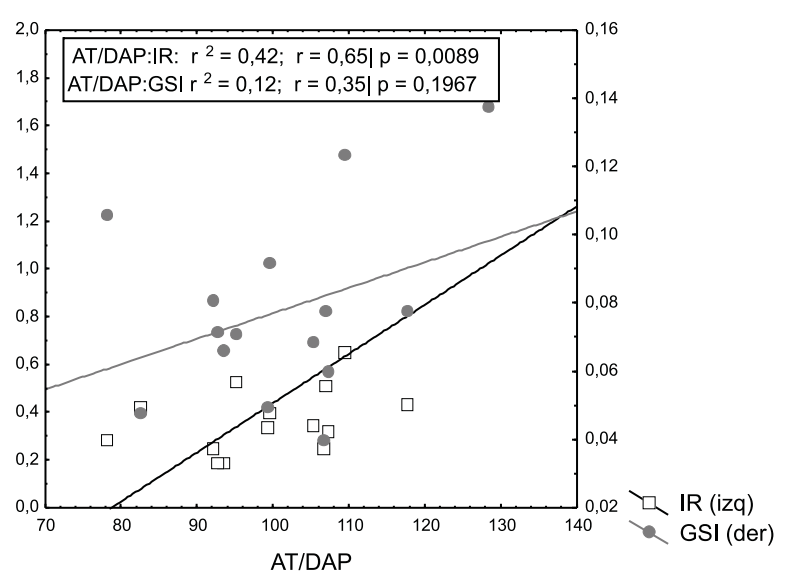

Figura 2 - Asociación entre IR y GSI con la relación entre altura total y diámetro de los árboles (AT/DAP). El modelo de regresión fue significativo solamente para IR.

Figura 2 - Associação entre IR e GSI com relação entre altura total e diâmetro de árvores $(A T / D A P)$. O modelo de regressão foi significativo apenas para o IR.

Las relaciones entre tensiones y las variables anatómicas cuantitativas no fueron significativas en ningún caso $(\mathrm{p}>0,05)$.

\section{DISCUSIÓN}

Las procedencias no tuvieron efecto sobre las tensiones de crecimiento

Hernández (2012) presentó, en un trabajo anterior y sobre un mayor número de árboles de este mismo ensayo $(n=45)$, que las procedencias tampoco fueron fuente significativa de variación. Contrariamente, Murphy et al. (2005) hallaron que la procedencia fue una fuente

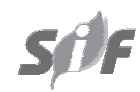

Revista Árvore, Viçosa-MG, v.38, n.4, p.755-763, 2014 


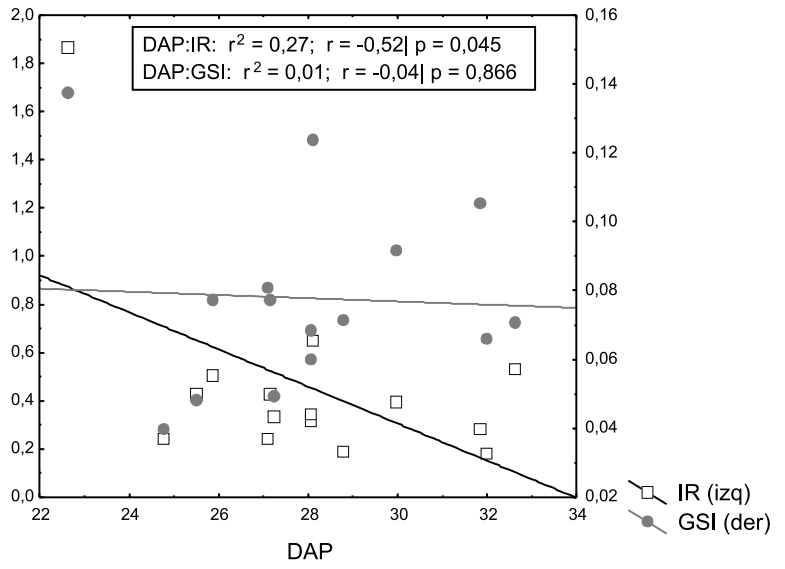

Figura 3 - Asociación entre IR y GSI con el diámetro de los árboles (DAP). El modelo de regresión fue significativo solamente para IR.

Figura 3-Associação entre IR e GSI com diâmetro da árvore (DAP). O modelo de regressão foi significativo apenas para oIR.

significativa de variación sobre las tensiones de crecimiento al estudiar 164 árboles de 9 años de $E$. dunni de 15 procedencias. Asimismo, demostraron que las familias involucradas dentro de las procedencias influyeron en las tensiones de crecimiento. Determinaron una heredabilidad intermedia a alta (0.3-0.5) para las tensiones lo que supone una interesante opción de manejo genético para reducir las tensiones.

\subsection{Anatomía de la madera}

Los caracteres anatómicos cuantitativos medidos son similares a los hallados en la bibliografía para la especie a esta edad y para otras especies del mismo género implantadas en Argentina (VILLEGAS; RIVERA, 2002; MONTEOLIVA et al., 2005). Los valores del espesor de pared de las fibras son un poco elevados con respecto a los valores reportados para la especie y para la densidad básica de $0,567 \mathrm{~g} . \mathrm{cm}^{-3}$ informada para estos árboles (HERNÁNDEZ, 2012). Esto puede deberse a la presencia de leño de tensión que tiende a engrosar las paredes de las fibras pero sin llegar a definirse una capa gelatinosa G característica de este leño (OKUYAMA et al., 1993; YOSHIDA et al., 2000, 2002; CLAIR et al., 2003; FANG et al., 2008). La ausencia de la capa $G$ fue informada para el género por varios autores y para otras angiospermas (BAILLERES et al., 1995; CLAIR et al., 2006; RUELLE et al., 2006). Sin embargo, según estos autores, pese a la ausencia de la capa $\mathrm{G}$ las tensiones de crecimiento son más elevadas en estas porciones de leño ya que se caracterizan por otras modificaciones químicas y estructurales (CLAIR et al., 2003, 2006; FANG et al., 2008). En estos trabajos la confirmación de la presencia de este tipo de leño no se puede realizar por observación de la anatomía clásica, por lo tanto se realiza indirectamente a través de la posición relativa en el tronco o rama con respecto al eje vertical, o de la medición de contenido de celulosa y lignina o ángulo microfibrillar (BAILLERES et al., 1995; CLAIR et al., 2006; RUELLE et al., 2006). Estas determinaciones no se realizaron en nuestro material.

\subsection{Tensiones de crecimiento}

Los resultados de las tensiones de crecimiento, medidos como GSI e IR, mostraron la variabilidad característica que se presenta con las tensiones en la bibliografía. En el trabajo anterior citado de los mismos autores (HERNÁNDEZ, 2012), la variabilidad hallada en las tensiones de crecimiento fue similar, al igual que el valor medio (GSI: 0,076 0,025 mm e IR: 0,43 $0,31 \%, n=45)$.

En comparación a otros trabajos realizados en el género Eucalyptus, los valores de tensiones de crecimiento fueron inferiores a los encontrados en la literatura. Para GSI en 20 árboles de E. dunnii de 14 años de edad se registró un promedio de $0,141 \mathrm{~mm}$ con un coeficiente de variación de 26,87\% (Souza, 2006), lo cual representa el doble que los hallados en nuestro trabajo. Otro estudio mostró en 16 árboles de E. dunnii de 19 años un promedio de 0,123 mm con un CV de 35,88\% (TRUGILHO; OLIVERA, 2008). Estos reportes demuestran valores notablemente más altos que la media hallada en nuestro trabajo y la gran dispersión de datos característica de las tensiones de crecimiento. Los magnitudes de IR fueron menores (aproximadamente la mitad) a los que se registraron en la especie $E$. grandis en condiciones experimentales equivalentes. En estudios realizados con el mismo índice de rajado utilizado en este trabajo se encontró que para plantaciones de 21 años de edad en rollizos de 3 metros de longitud obtenidos a partir del 1,7 m de altura, los valores promedio de IR variaron entre $0,67 \%$ y $0,87 \%$ según el tratamiento silvicultural aplicado (LIMA, 2005). En cuanto a la variación del IR, los valores se comportaron en forma similar a los estudios existentes, como el llevado a cabo por Malan (2008) que obtuvo un CV de 88,58\% en una investigación de que involucró la evaluación de 121 árboles de $E$. grandis de 21 años. Bajo este contexto 
bibliográfico, podría considerarse que estas 3 procedencias y en el marco de las limitaciones que presupone un único ensayo, el E. dunnii creciendo en estas condiciones desarrolla tensiones de crecimiento bajas. Lo cual implica un alto rendimiento en tablas en el aserrado y pocos defectos de grietas y deformaciones (datos no mostrados, HERNÁNDEZ, 2012).

\subsection{Relaciones entre variables}

Sobre las relaciones halladas entre las tensiones de crecimiento y las variables dendrométricas de los árboles, la bibliografía indica resultados contradictorios. Así, Murphy et al (2005) no encontraron correlación entre las tensiones de crecimiento y el DAP en 164 árboles de E. dunnii de 9 años, pero si para la relación entre altura/DAP. En contraste Trujilho et al. (2008) hallaron una relación positiva entre DAP y GSI para esta especie en plantaciones de 15 y 19 años. Malan (2008) mostró que no hubo relación entre el IR y el DAP en E. grandis de 20 años implantado en Sudáfrica. En E. globulus, Vignote et al. (1996) han publicado una relación entre edad, DAP, y AT/DAP, entre otras variables, para 165 árboles implantados en 4 sitios de España. Sin embargo, el conjunto de las variables entre las que se incluye el tamaño de los árboles solo llega a explicar el 18\% de la variación hallada en las tensiones de crecimiento medidas como GSI. La edad explica por sí sola el 13\% de la variación. Valencia et al. (2011) encontraron relación entre IR y DAP en 81 árboles de $E$. nitens de 22 años en un ensayo de raleo en Tasmania, pero no lo hicieron para GSI. Del análisis bibliográfico expuesto se desprende que la variabilidad hallada es muy alta, por lo tanto estas relaciones deberían testearse en un mayor número de árboles, edades, sitios y tratamientos silvícolas.

Las relaciones entre tensiones y las variables anatómicas cuantitativas determinadas en este trabajo, no fueron significativas en ningún caso $(p>0,05)$. Sin embargo, se han reportado vinculaciones entre las tensiones y el diámetro y frecuencia de vasos (RUELLE et al., 2006; GACITUA et al., 2007). Un trabajo sobre $E$. nitens reportó que la frecuencia de vasos estaba afectando las tensiones de crecimiento sin importar el diámetro de los mismos. Desde el punto de vista mecánico, los vasos se consideran una interrupción en el plan estructural de un sólido, en este caso madera, y por lo tanto estos vasos son el sitio de plano de falla que resiste en menor medida el esfuerzo de las tensiones (GACITUA et al., 2007). Ruelle et al. (2006) en un trabajo sobre anatomía del leño de tensión de 21 especies de latifoliadas tropicales, publicaron que dentro de los parámetros cuantitativos relativos a fibras y vasos, la frecuencia de vasos fue la característica que se correlacionó negativamente a las tensiones de crecimiento medidas. Otras variables como forma y superficie de los vasos, ancho y espesor de pared de las fibras no revelaron ninguna tendencia general. En nuestro trabajo, la relación más alta hallada entre tensiones y variables anatómicas, fue precisamente GSI y frecuencia de vasos con una correlación de 0,41 marginalmente significativa $(\mathrm{p}=0,067)$.

Las relaciones más importantes halladas en la literatura entre tensiones y propiedades anatómicas revelan la importancia de la formación de leño de tensión en el aumento de las tensiones sufridas en la madera, con presencia o no de la capa gelatinosa G (OKUYAMA et al., 1993; BAILLERES et al., 1995; CLAIR et al., 2003, 2006; FANG et al., 2008). En un trabajo posterior, se debería determinar la presencia de leño de tensión sobre estas muestras, ya que el elevado espesor de pared hallado en las fibras y la forma redondeada de las mismas permite inferir la presencia de este tipo de leño.

\section{CONCLUSIONES}

Las procedencias no afectaron el tamaño de los árboles, las propiedades anatómicas ni las tensiones de crecimiento, por lo tanto puede utilizarse indistintamente este material genético en plantaciones comerciales para un destino de aserrado.

Las tensiones de crecimiento medidas sobre este material genético y estas condiciones de cultivo fueron bajas, lo que implicaría un alto rendimiento en tablas en el aserrado y pocos defectos de grietas y deformaciones.

Dentro de las variables de los árboles solo el DAP y la relación AT/DAP tuvieron una relación significativa y baja con las tensiones de crecimiento medidas como IR. Estos resultados permiten explorar/aplicar diferentes tratamientos silvícolas de aumento de productividad y volumen de los árboles con cierta libertad sin esperar un gran aumento de las tensiones en el fuste.

Las relaciones entre tensiones y las variables anatómicas cuantitativas no fueron significativas en ningún caso, por lo tanto puede esperarse que ciertas

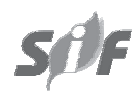

Revista Árvore, Viçosa-MG, v.38, n.4, p.755-763, 2014 
decisiones silvícolas, que puedan afectar la calidad de la madera producida en términos anatómicos, no tengan grandes repercusiones sobre las tensiones. Sin embargo, en futuras investigaciones debe indagarse, sobre la formación, presencia y medición de leño de tensión asociado a las tensiones de crecimiento sobre este material.

\section{REFERÊNCIAS}

BAILLERES, H. et al. Structure, composition chimique et retraits de maturation du bois chez les clones d'Eucalyptus. Annals Forest Science, v.52, n.1, p.157-172, 1995.

CAstro, G. O. et al. Caracterización agroclimática de la Provincia de Corrientes. 1ra y 2da Etapa. Buenos Aires: Consejo Federal de Inversiones, 1991. p.237.

CLAIR, B.; RUELLE, J.; THIBAUT, B. Relationship between growth stresses, mechano-physical properties and proportion of fibre with gelatinous layer in chestnut (Castanea Sativa Mill.). Holzforschung, v.57, n.1, p.189-195, 2003.

CLAIR, B. et al. Tension wood and opposite wood in 21 tropical rain forest species 1 . Occurrence and efficiency of the G-layer. IAWA Journal, v.27, n.3, p.329-338, 2006.

DICKSON, R. L. et al. Segregation of Eucalyptus dunnii logs using acoustics. Forest Ecology and Management, v.179, p.243-251, 2003.

FANG, CH-Q. et al. Growth stresses are highly controlled by the Amount of g-layer in poplar tension wood. IAWA Journal, v.29, n.3, p.237246,2008

GACITUA, W. et al. Nanoindentaciones y ultraestructura en madera de Eucalyptus nitens con micro y meso grietas. Maderas. Ciencia y Tecnología, v.9, n.3, p.259-270, 2007.

Gerard, J.; ThiBAut, B. (Ed.) Actes des séminaires annuels du programme Architecture, structure, mecanique de l’arbre. Paris: Université de Montpellier II, Sciences et Techniques du Languedoc, 1 er décembre, 1992.
GERARD, J. Constraintes de croissance, variations internes de densite et de module d' elasticote longitudinal, et deformations de sciage chez les eucalyptus de plantation. Thèse (Docteur) - L’ Universite de Bordeaux I, 1994.

HERNÁNDEZ, M. Efecto de las tensiones de crecimiento en la madera de Eucalyptus dunnii Maiden (Myrtaceae). Tesis (Maestría) - Universidad Nacional de Misiones, 2012.

KUBLER, H. Growth stresses in trees and related wood properties. Forestry Abstracts, v.48, n.3, p.131-189, 1987.

LIMA, I. L. Influência do desbaste e da adubação na qualidade da madeira serrada de Eucalyptus grandis Hill exMaiden. 2005. 137f. Tese (Doutorado em Engenharia Florestal) - Escola Superior de Agricultura Luiz de Queiroz, Piracicaba, 2005.

MALAN, F. S. Clonal differences in log end splitting in Eucalyptus grandis in relation to age, parent performance, growth rate and wood density in two even-aged trials in Mpumalanga, South Africa. Southern Forests, v.70, n.1, p.37-43, 2008.

MONTEOLIVA, S.; ÁLVAREZ, J.; IGARTÚA, D. Evolución radial de la morfología fibrosa y la densidad básica en la madera de Eucalyptus globulus. ACTAS DEL TERCER CONGRESO FORESTALARGENTINO Y LATINOAMERICANO, Corrientes, Argentina, 2005.

MURPHY, T.; HENSON, M.; VANCLAY, J. K. Growth stress in Eucalyptus dunnii. Australian Forestry, v.68, n.2, p.144-149, 2005.

OKUYAMA, T. et al. Growth stresses in wood tension: role of microfibrils and lignifications. Annals Forest Science, v.51, p.291-300, 1993.

PEDROSA, M. Performance de vigas “I” constiuídas por flanges de PLP y almas de compensado de Pinus taeda $L$ y Eucalyptus dunnii Maiden, e alma de OSB de Pinus spp. 2003. 106.. Tese (Mestrado em Engenharia Florestal) - Universidade Federal do Paraná, Curitiba, 2003. 
PEREYRA, O. Evaluación de la madera de Eucalyptus dunnii (Maid) en la producción de láminas. Yvyraretá, v. 10, n.1, p.15-20, 2000.

RAYMOND, C. A. et al. Evaluation of nondestructive methods of measuring growth stress in Eucalyptus globulus: relationships between strain, wood properties and stress. Forest Ecology and Management, v.190, p.187200, 2004.

RUELLE, J. et al. Tension wood and opposite wood in 21 tropical rain forest species. IAWA Journal, v.27, n.4, p.341-376, 2006.

SEVERO, E.; TOMASELLI, I. Efeito da prévaporização em algumas propriedades físicas da madeira de Eucalyptus dunnii. Cerne, v.7, n.2, p.35-42, 2001.

SOUZA, M.A.M. Metodologias não destrutivas para avaliação das tensões de crescimento em Eucalyptus dunnii Maiden. 2006. 90f. Tese (Doutorado em Engenharia Florestal) - Universidade Federal do Paraná, Curitiba, 2006.

THIBAUT, B.; GRIL, J. Growth stresses. In: BARNET, J.R.; JERONIMIDIS, G. (Ed.) Wood quality and its biological basis. Oxford: Blackwell Publishing, 2003.

TRUGILHO, P. F.; OLIVERA, J. T. S. Relações e estimativas da tensão de crescimento longitudinal em Eucalyptus dunnii Maiden. Revista Arvore, v.32, n.4, p.723-729, 2008.
VALENCIA, J. et al. Longitudinal growth strain as a log and wood quality predictor for plantation-grown Eucalyptus nitens sawlogs. Wood Science and Technology, v.45, n.1, p.15-34, 2011.

VILLEGAS, M. S.; RIVERA, S. M. Revisión xilológica de las principales especies del género Eucalyptus L'Herit. cultivadas en Argentina. Revista da Faculdade de Agrononia, v.105, n.1, p.9-28, 2002.

VIGNOTE, S. et al. Estudio de las tensiones de crecimiento de Eucalyptus globulus Labill en Galicia y su relación con las características de la estación y morfológicas del propio árbol.

Investigación Agraria Sistemas y Recursos Forestales, v.5, n.1, p.153-176, 1996.

YANG, J. L. et al. Measurement methods for longitudinal surface strain in trees: a review. Australian Forestry, v.68, n.1, p.34-43, 2005.

YANG, J. L. et al. The effect of growth strain and other defects on the sawn timber quality of 10 year-old Eucalyptus globulus Labill.

Australian Forestry, v.65, n.1, p.31-37, 2002.

YOSHIDA, M.; OKUDA, T.; OKUYAMA, T. Tension wood and growth stress induced by artificial inclination in Liriodendron tulipifera Linn. and Prunus spachiana Kitamura f. ascendens Kitamura. Annals Forest Science., v.57, p.739-746, 2000.

YOSHIDA, M. et al. Tensile growth stress and lignin distribution in the cell walls of yellow poplar, Liriodendron tulipifera Linn. Trees, v.16, p.457-464, 2002. 
